\title{
On the closure of Baire classes under transfinite convergences
}

by

\author{
Tamás Mátrai (Budapest)
}

\begin{abstract}
Let $X$ be a Polish space and $Y$ be a separable metric space. For a fixed $\xi<\omega_{1}$, consider a family $f_{\alpha}: X \rightarrow Y\left(\alpha<\omega_{1}\right)$ of Baire- $\xi$ functions. Answering a question of Tomasz Natkaniec, we show that if for a function $f: X \rightarrow Y$, the set $\left\{\alpha<\omega_{1}: f_{\alpha}(x) \neq\right.$ $f(x)\}$ is finite for every $x \in X$, then $f$ itself is necessarily Baire- $\xi$. The proof is based on a characterization of $\Sigma_{\eta}^{0}$ sets which can be interesting in its own right.
\end{abstract}

1. Introduction. It is a fact of life that the class of continuous real functions is not closed under pointwise convergence: instead, we obtain a realization of the Baire- 1 functions. On the other hand, it is an easy exercise that the pointwise limit of a sequence of continuous functions with length $\omega_{1}$ is necessarily continuous.

This problem and other properties of the pointwise convergence of transfinite sequences of real functions have been first considered by W. Sierpiński [7]. In particular, he studied which class of functions is closed under such convergences. Since most of the classes, for example the class of Baire- $\xi$ functions for $\xi \geq 2$, are not, T. Natkaniec [6] introduced a stronger notion of pointwise convergence. We recall the precise setting in the following definition.

Definition 1. Let $\lambda$ be a cardinal, $(X, \tau)$ be a Polish space, $(Y, d)$ be a separable metric space, and consider an ideal $\mathcal{I}$ on $\lambda$. We say that a sequence of functions $f_{\alpha}: X \rightarrow Y(\alpha<\lambda) \mathcal{I}$-converges to the function $f: X \rightarrow Y$, in notation $f_{\alpha} \rightarrow \mathcal{I} f$, if

$$
\left\{\alpha<\lambda: f_{\alpha}(x) \neq f(x)\right\} \in \mathcal{I}
$$

for every $x \in X$.

2000 Mathematics Subject Classification: Primary 26A21.

This research was carried out while the author was visiting the Équipe d'Analyse Fonctionnelle de l'Université Pierre et Marie Curie - Paris 6 in the Analysis and Operators European Training Network. The research was partially supported by the OTKA grant F 043620 . 
Similarly, we write $f_{\alpha} \rightarrow_{\mathcal{I}} f$ if for every $\varepsilon>0$ and $x \in X$ we have

$$
\left\{\alpha<\lambda: d\left(f(x), f_{\alpha}(x)\right)>\varepsilon\right\} \in \mathcal{I} .
$$

In the case of the ordinary $\omega_{1}$ convergence, as used in [3] and [7], we have $\lambda=\omega_{1}$ and $\mathcal{I}=\left[\omega_{1}\right]^{\leq \omega}$, that is, the ideal of countable subsets of $\omega_{1}$. However, our motivating theorem, answering [6, Problem 1, p. 490], is related to the particular case when the ideal consists of the finite subsets of $\omega_{1}$, that is, $\mathcal{I}_{<}=\left[\omega_{1}\right]^{<\omega}$.

Theorem 2. Let $(X, \tau)$ be a Polish space, $(Y, d)$ be a separable metric space, and for a fixed $\xi<\omega_{1}$ consider a family $f_{\alpha}: X \rightarrow Y\left(\alpha<\omega_{1}\right)$ of Baire- $\xi$ functions. If $f: X \rightarrow Y$ is such that $f_{\alpha} \rightarrow_{\mathcal{I}_{<}} f$, then $f$ is Baire- $\xi$.

We note here that the original question asked by $\mathrm{T}$. Natkaniec referred

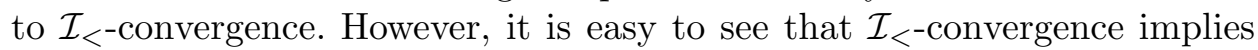
${ }_{\mathcal{I}}^{d}$-convergence, so the result above is formally stronger than required. The sufficiency of ${ }_{\mathcal{I}_{<}}^{d}$-convergence was pointed out to the author by Petr Holický.

As shown by W. Sierpiński ([7, Theorem 1, p. 133 and Theorem 2, p. 137]), for the class of continuous and Baire-1 functions Theorem 2 also holds for $\mathcal{I}=\left[\omega_{1}\right]^{\leq \omega}$ instead of $\mathcal{I}_{<}$. On the other hand, it is independent for every $2 \leq \xi<\omega_{1}$ whether there is an $\left[\omega_{1}\right] \leq \omega_{\text {-convergent sequence of }}$ Baire- $\xi$ functions whose limit function is Borel but not Baire- $\xi$ (observe that

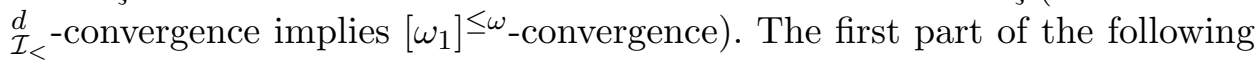
theorem has already been proved by W. Sierpiński ([7, Section 6, pp. 139 and 140]) and further discussed by P. Komjáth ([3, Theorem 3, p. 499]). Its second part, related to Problem 3 in [6, p. 490], is a simple analogue of Theorem 2.

Theorem 3. Let $(X, \tau)$ be a Polish space and $(Y, d)$ be a separable metric space.

(i) (W. Sierpiński, P. Komjáth). Assuming the continuum hypothesis, there exists an $\left[\omega_{1}\right]^{\leq \omega}$-convergent sequence of real Baire-2 functions whose limit function is not Borel.

(ii) Let $\lambda<2^{\aleph_{0}}$ be an infinite cardinal with $\operatorname{cf}(\lambda)>\omega$ and set $\mathcal{J}=$ $[\lambda]^{<\lambda}$. For a fixed $\xi<\omega_{1}$, consider a family $f_{\alpha}: X \rightarrow Y(\alpha<\lambda)$ of Baire- $\xi$ functions and a Borel function $f: X \rightarrow Y$. If $f_{\alpha} \rightarrow_{\mathcal{J}} f$ and in our model the union of $\lambda$ meager sets is meager in Polish spaces, then $f$ is necessarily Baire- $\xi$.

The assumption on the additivity of meager sets holds under $\mathrm{MA}(\lambda)$ (see e.g. [1, Theorem 1.2, p. 505] or [5, Theorem, p. 170]). The convergence of transfinite sequences of Baire-2 functions of length $\omega_{2}$ has also been investigated by P. Komjáth (see [3, Theorems 4 and 5, p. 500]). It is consistent (with $2^{\aleph_{0}}=\omega_{2}$ and $\mathrm{MA}\left(\omega_{1}\right)$ ) that every real function can be obtained as 
such a limit. It is also consistent, under more complicated assumptions, that the limit function is necessarily Baire-2.

It is not surprising, and we will follow this direction, that the proofs of Theorem 2 and Theorem 3(ii) go via the analogous statements for characteristic functions, i.e. for sets of given Borel classes. As usual, $\Pi_{\xi}^{0}(\tau)\left(\Sigma_{\xi}^{0}(\tau)\right.$ resp.) stands for the $\xi$ th multiplicative (additive resp.) Borel class in $(X, \tau)$, starting with $\Pi_{1}^{0}(\tau)=$ closed sets, $\Sigma_{1}^{0}(\tau)=$ open sets. With this notation our key lemma, which might be considered as the main result of this paper, can be stated as follows.

TheOrem 4. Let $\tau_{C_{1}}$ denote the product topology on $2^{\omega}$; let $(X, \tau)$ be a Polish space. For every $2 \leq \xi<\omega_{1}$, there exist a $\Pi_{\xi}^{0}\left(\tau_{C_{1}}\right)$ set $P_{\xi} \subseteq 2^{\omega}$ and a Polish topology $\tau_{\xi}$ on $2^{\omega}$ which is finer than $\tau_{C_{1}}$ such that $P_{\xi}$ is nowhere dense and closed in the topology $\tau_{\xi}$, and if a Borel set $A \subseteq X$ is

(i) in $\Sigma_{\xi}^{0}(\tau)$, then whenever for a continuous one-to-one mapping $\varphi:\left(2^{\omega}, \tau_{C_{1}}\right) \rightarrow(X, \tau)$ the set $\varphi^{-1}(A) \cap P_{\xi}$ is of second category in $P_{\xi}$ in the relative topology $\left.\tau_{\xi}\right|_{P_{\xi}}$, then $\varphi^{-1}(A) \subseteq 2^{\omega}$ is of second category in the topology $\tau_{\xi}$;

(ii) not in $\Sigma_{\xi}^{0}(\tau)$, then there is a continuous one-to-one mapping $\varphi:\left(2^{\omega}, \tau_{C_{1}}\right) \rightarrow(X, \tau)$ such that $\varphi\left(P_{\xi}\right) \subseteq A$ and $\varphi^{-1}(A) \subseteq 2^{\omega}$ is of first category in the topology $\tau_{\xi}$.

Moreover, if $\lambda<2^{\aleph_{0}}$ is a cardinal and in our model the union of $\lambda$ meager sets is meager in Polish spaces, then the first statement holds for every (not necessarily Borel) set $A$ which can be obtained as a union of $\lambda$ many $\Sigma_{\xi}^{0}(\tau)$ sets.

Informally, this theorem says that a fixed proper $\Pi_{\xi}^{0}(\tau)$ set is so far from being a $\Sigma_{\xi}^{0}(\tau)$ set that even Baire category can distinguish them in a suitable topology (a similar result was obtained by S. Solecki in $[8$, Theorem 2.2 , p. 526]). This approach explains the appearance of the condition on the additivity of meager sets in Theorem 3(ii). The last statement is necessary to prove Theorem 3(ii), and in other words it states that our assumption on the additivity of meager sets implies that if the union of $\lambda$ many $\Sigma_{\xi}^{0}\left(\tau_{C_{1}}\right)$ sets is Borel, then it is $\Sigma_{\xi}^{0}\left(\tau_{C_{1}}\right)$ (see also [8, Corollary 2.3, p. 526] and [9]).

Theorem 4 can also be regarded as a qualitative analogue of the following result (see e.g. [4, p. 433] for the $\xi \geq 3$ case and [2, Theorem 21.22, p. 161] for the $\xi=2$ case), that we will use in the proof.

Theorem 5 (A. Louveau, J. Saint Raymond). Let $3 \leq \xi<\omega_{1}$ and $(X, \tau)$ be a Polish space. If $P_{\xi} \subseteq 2^{\omega}$ is $\Pi_{\xi}^{0}\left(\tau_{C_{1}}\right)$ but not $\Sigma_{\xi}^{0}\left(\tau_{C_{1}}\right)$ and $A_{0}, A_{1} \subseteq X$ is any pair of disjoint Borel sets, then either $A_{0}$ can be separated from $A_{1}$ by a $\Sigma_{\xi}^{0}(\tau)$ set or there is a continuous one-to-one map $\varphi:\left(2^{\omega}, \tau_{C_{1}}\right) \rightarrow X$ with 
$\varphi\left(P_{\xi}\right) \subseteq A_{0}$ and $\varphi\left(2^{\omega} \backslash P_{\xi}\right) \subseteq A_{1}$. The same conclusion holds for $\xi=2$ if $P_{2} \subseteq 2^{\omega}$ is the complement of a dense countable set.

Our reference for the basic notions of descriptive set theory is [2]. In the next section we prove Theorems 2 and 3(ii), while the proof of Theorem 4 will be given in the last section.

2. I-convergent functions. In order to establish the connection between function classes and sublevel sets we will use the following classical result (see e.g. [2, Chapter II, Theorem 24.3, p. 190]).

Theorem 6. Let $(X, \tau)$ be a Polish space and $(Y, d)$ be a separable metric space. Then for every $1 \leq \xi<\omega_{1}$, a function $f: X \rightarrow Y$ is Baire- $\xi$ if and only if $f^{-1}(U) \subseteq X$ is $\Sigma_{\xi+1}^{0}(\tau)$ for every open set $U \subseteq Y$.

In the metric space $(Y, d)$, the open ball centered at $x \in Y$ with radius $\rho$ is denoted by $B_{d}(x, \rho)$. After these preparations, Theorems 2 and 3(ii) are simple corollaries of Theorem 4 .

Proof of Theorem 2. By [7, Theorem 1, p. 133 and Theorem 2, p. 137], the statement holds for $\xi \leq 1$. So fix $2 \leq \xi<\omega_{1}$ and suppose that $f_{\alpha} \rightarrow_{\mathcal{I}_{<}} f$ for a family $f_{\alpha}: X \rightarrow Y\left(\alpha<\omega_{1}\right)$ of Baire- $\xi$ functions.

Suppose that $f$ is not Baire- $\xi$. Being the pointwise limit of $\left\{f_{\alpha}: \alpha<\omega\right\}$, $f$ is clearly Borel, so by Theorem 6 , there is an open ball $B_{d}(x, \rho) \subseteq Y$ such that $f^{-1}\left(B_{d}(x, \rho)\right)$ is Borel but not $\Sigma_{\xi+1}^{0}(\tau)$. Set

$$
H(\varepsilon)=f^{-1}\left(B_{d}(x, \rho-\varepsilon)\right), \quad H_{\alpha}(\varepsilon)=f_{\alpha}^{-1}\left(B_{d}(x, \rho-\varepsilon)\right)
$$

for every $\alpha<\omega_{1}$ and $0<\varepsilon<\varrho$. Note that by Theorem $6, H_{\alpha}(\varepsilon)$ is in $\Sigma_{\xi+1}^{0}(\tau)$ for every $\alpha<\omega_{1}$ and $0<\varepsilon<\varrho$.

Since $H(0)$ is not $\Sigma_{\xi+1}^{0}(\tau)$, by Theorem 4(ii) there is a continuous oneto-one map $\varphi:\left(2^{\omega}, \tau_{C_{1}}\right) \rightarrow(X, \tau)$ such that

(i) $\varphi\left(P_{\xi+1}\right) \subseteq H(0)$,

(ii) $\varphi^{-1}(H(0)) \subseteq 2^{\omega}$ is of first category in the topology $\tau_{\xi+1}$.

By (i), there is an $\varepsilon_{0}>0$ such that $\varphi^{-1}\left(H\left(\varepsilon_{0}\right)\right) \cap P_{\xi+1}$ is of second category in the topology $\left.\tau_{\xi+1}\right|_{P_{\xi+1}}$. Let $J_{1}(\varepsilon)$ denote the set of those $\alpha<\omega_{1}$ for which $\varphi^{-1}\left(H_{\alpha}(\varepsilon)\right)$ is of second category in the topology $\tau_{\xi+1}$.

We prove that $\omega_{1} \backslash J_{1}(\varepsilon)$ is finite for every $\varepsilon<\varepsilon_{0}$. Suppose that this is not true and take a countably infinite set $J^{\prime}(\varepsilon) \subseteq \omega_{1} \backslash J_{1}(\varepsilon)$. By the definition of $\underset{\mathcal{I}_{<}}{d}$-convergence, $\varepsilon<\varepsilon_{0}$ implies that

$$
H\left(\varepsilon_{0}\right) \subseteq H^{\prime}(\varepsilon):=\bigcup_{\alpha \in J^{\prime}(\varepsilon)} H_{\alpha}(\varepsilon)
$$

so $\varphi^{-1}\left(H^{\prime}(\varepsilon)\right) \cap P_{\xi+1}$ is of second category in $P_{\xi+1}$ in the topology $\left.\tau_{\xi+1}\right|_{P_{\xi+1}}$; 
that is, since $H^{\prime}(\varepsilon)$ is $\Sigma_{\xi+1}^{0}(\tau)$, by Theorem $4.1, \varphi^{-1}\left(H^{\prime}(\varepsilon)\right)$ is of second category in $\tau_{\xi+1}$. This is a contradiction, since by the definition of $J_{1}(\varepsilon)$, $\varphi^{-1}\left(H^{\prime}(\varepsilon)\right)$ is $\tau_{\xi+1}$-meager.

So $J_{1}(\varepsilon)$ is of cardinality $\omega_{1}$ for every $\varepsilon<\varepsilon_{0}$. In particular, given that $\left(2^{\omega}, \tau_{\xi+1}\right)$ has countable base, there is a $\tau_{\xi+1}$-open set $U \subseteq 2^{\omega}$ such that for a countably infinite set $J^{\prime \prime} \subseteq J_{1}\left(\varepsilon_{0} / 2\right)$ the set $\varphi^{-1}\left(H_{\alpha}\left(\varepsilon_{0} / 2\right)\right)$ is residual in $U$ in the topology $\tau_{\xi+1}$ whenever $\alpha \in J^{\prime \prime}$. Hence for

$$
H^{\prime \prime}=\bigcap_{\alpha \in J^{\prime \prime}} H_{\alpha}\left(\varepsilon_{0} / 2\right)
$$

$\varphi^{-1}\left(H^{\prime \prime}\right)$ is also $\tau_{\xi+1}$-residual in $U$, so by (ii) we can find a point $x_{0} \in$ $H^{\prime \prime} \backslash H(0)$. Thus $f_{\alpha}\left(\alpha<\omega_{1}\right)$ is not $\stackrel{\mathcal{I}}{<}_{<}$-convergent since

$$
J^{\prime \prime} \subseteq\left\{\alpha<\omega_{1}: d\left(f\left(x_{0}\right), f_{\alpha}\left(x_{0}\right)\right)>\varepsilon_{0} / 2\right\}
$$

is infinite; a contradiction. The proof is complete.

Proof of Theorem 3(ii). Again, for $\xi \leq 1$ the statement follows from the proofs in [7]; so let $\xi \geq 2$. Now $f$ is Borel by assumption; and the proof is the same as for Theorem 2, until the definition of $J_{1}$. Now we show that $\operatorname{card}\left(\lambda \backslash J_{1}(\varepsilon)\right)<\lambda$ for every $\varepsilon<\varepsilon_{0}$.

Suppose that this is not true and take a set $J^{\prime}(\varepsilon) \subseteq \lambda \backslash J_{1}(\varepsilon)$ of cardinality $\lambda$. By the definition of ${ }_{\mathcal{J}}^{d}$-convergence, $\varepsilon<\varepsilon_{0}$ implies that

$$
H\left(\varepsilon_{0}\right) \subseteq H^{\prime}(\varepsilon):=\bigcup_{\alpha \in J^{\prime}(\varepsilon)} H_{\alpha}(\varepsilon),
$$

so $\varphi^{-1}\left(H^{\prime}(\varepsilon)\right) \cap P_{\xi+1}$ is of second category in $P_{\xi+1}$ in the topology $\left.\tau_{\xi+1}\right|_{P_{\xi+1}}$; that is, by the extension of Theorem $4(\mathrm{i})$, since $H^{\prime}(\varepsilon)$ is the union of $\lambda$ many $\Sigma_{\xi+1}^{0}(\tau)$ sets $H_{\alpha}(\varepsilon)\left(\alpha \in J^{\prime}(\varepsilon)\right), \varphi^{-1}\left(H^{\prime}(\varepsilon)\right)$ is of second category in $\tau_{\xi+1}$. Now this contradicts the assumption that the union of $\lambda$ meager sets is meager in $\left(2^{\omega}, \tau_{\xi+1}\right)$, since by the definition of $J_{1}(\varepsilon), \varphi^{-1}\left(H_{\alpha}(\varepsilon)\right)\left(\alpha \in J^{\prime}(\varepsilon)\right)$ is $\tau_{\xi+1}$-meager.

We continue as above; $J_{1}(\varepsilon)$ is of cardinality $\lambda$ for every $\varepsilon<\varepsilon_{0}$. In particular, given that $\operatorname{cf}(\lambda)>\omega$ and $\left(2^{\omega}, \tau_{\xi+1}\right)$ has countable base, there is a $\tau_{\xi+1}$-open set $U \subseteq 2^{\omega}$ such that for a set $J^{\prime \prime} \subseteq J_{1}\left(\varepsilon_{0} / 2\right)$ of cardinality $\lambda$ the set $\varphi^{-1}\left(H_{\alpha}\left(\varepsilon_{0} / 2\right)\right)$ is $\tau_{\xi+1}$-residual in $U$ whenever $\alpha \in J^{\prime \prime}$. Since in our model the intersection of $\lambda$ many $\tau_{\xi+1}$-residual sets is again residual, for

$$
H^{\prime \prime}=\bigcap_{\alpha \in J^{\prime \prime}} H_{\alpha}\left(\varepsilon_{0} / 2\right)
$$

$\varphi^{-1}\left(H^{\prime \prime}\right)$ is also $\tau_{\xi+1}$-residual in $U$, so by (ii) we can find a point $x_{0} \in H^{\prime \prime} \backslash$ $H(0)$. Again, this contradicts the ${ }_{\mathcal{J}}^{d}$-convergence. The proof is complete. 
3. Distinguishing Borel classes. We will define recursively a sequence of compact Polish spaces homeomorphic to $\left(2^{\omega}, \tau_{C_{1}}\right)$, Borel sets of increasing complexity and additional Polish topologies which will serve as test sets and topologies. During this construction we will successively refine Polish topologies by turning countably many pairwise disjoint closed sets into open sets. We do this as described in [2], that is, the open sets of the old topology together with their portions on the members of our collection of closed sets serve as a base of a new, finer topology. We will use the fact that the topology obtained this way is also Polish.

We will also need a precise notion of basic open sets in our resulting spaces. In what follows, if a basis $\mathcal{G}_{i}$ is fixed in the space $\left(X_{i}, \sigma_{i}\right)$ for every $i \in I$, which are meant to be the basic open sets in $X_{i}$, then the basic open sets of $\left(\prod_{i \in I} X_{i}, \prod_{i \in I} \sigma_{i}\right)$ are the open sets of the form

$$
\prod_{i \in J} G_{i} \times \prod_{i \in I \backslash J} X_{i}
$$

where $J \subseteq I$ is finite and $G_{i} \in \mathcal{G}_{i}$ for every $i \in J$. Similarly, if the basic open sets $\mathcal{G}$ are fixed in $(X, \sigma)$ and $\mathcal{F}$ is a countable collection of pairwise disjoint closed subsets of $X$, then the basic open sets of the finer topology obtained as described above are of the form $G \cap F$ or $G$ with $G \in \mathcal{G}, F \in \mathcal{F}$. Observe that the basic open sets thus defined form a basis.

We will have to return to the topologies on the coordinate spaces in product spaces. If $(X, \sigma),(Y, \tau)$ are arbitrary topological spaces and $(\mathcal{X}, \mathcal{S})=$ $(X \times Y, \sigma \times \tau)$, then we define $\operatorname{Pr}_{X}(\mathcal{S})=\sigma$. The projection of product sets in product spaces is defined analogously,

Now we can start the construction. We set $C_{1}=2^{\omega}$ and

$$
P_{1}=\left\{x \in C_{1}: \forall m \in \omega(x(m)=1)\right\} .
$$

We denote by $\tau_{C_{1}}$ the product topology on $C_{1}$; so $\left(C_{1}, \tau_{C_{1}}\right)$ is a Polish space. Set $\tau_{1}=\tau_{C_{1}}$ on $C_{1}$.

For every ordinal $\xi<\omega_{1}$ we fix once and for all a sequence

$$
\xi_{1} \leq \cdots \leq \xi_{i} \leq \ldots<\xi \quad(i<\omega)
$$

of ordinals: if $\xi$ is limit, let $\xi=\lim _{i \rightarrow \infty} \xi_{i}$, while for $\xi$ successor, $\xi=\xi_{i}+1$ for every $i<\omega$. To avoid complicated notations, we do not indicate the dependence of the sequence on $\xi$; it will always be clear which pair of an ordinal and a sequence is considered.

Suppose that the sets $C_{\eta}$ and $P_{\eta}$ and their topologies are defined for every $\eta<\xi$. Then let $C_{\xi}=\prod_{i=0}^{\infty} C_{\xi_{i}}$ and

$$
\begin{gathered}
P_{\xi}=\left\{x \in C_{\xi}: \forall m \in \omega\left(x(m, \cdot) \in C_{\xi_{m}} \backslash P_{\xi_{m}}\right)\right\}, \\
\tau_{C_{\xi}}=\prod_{i=0}^{\infty} \tau_{C_{\xi_{i}}}, \quad \tau_{\xi}^{<}=\prod_{i=0}^{\infty} \tau_{\xi_{i}},
\end{gathered}
$$


and let $\tau_{\xi}$ be the coarsest topology extending $\tau_{\xi}^{<}$such that

$$
\begin{aligned}
U_{\xi, N} & =\prod_{i=0}^{N-1}\left(C_{\xi_{i}} \backslash P_{\xi_{i}}\right) \times P_{\xi_{N}} \times \prod_{i=N+1}^{\infty} C_{\xi_{i}} \\
& \subseteq \prod_{i=0}^{N-1} C_{\xi_{i}} \times C_{\xi_{N}} \times \prod_{i=N+1}^{\infty} C_{\xi_{i}}=C_{\xi}
\end{aligned}
$$

is open for every $N<\omega$. It is important that the sets $U_{\xi, N}(N<\omega)$ are pairwise disjoint. Note also that this construction fits into the framework presented in the introduction of this section since after having turned $U_{\xi, i}$ into an open set for $i<N$,

$$
U_{\xi, N}=\left(\prod_{i=0}^{N-1} C_{\xi_{i}} \times P_{\xi_{N}} \times \prod_{i=N+1}^{\infty} C_{\xi_{i}}\right) \backslash\left(\bigcup_{i=0}^{N-1} U_{\xi, i}\right)
$$

is indeed closed in this intermediate refinement of $\tau_{\xi}^{<}$.

In the following six claims we prove some relations between $P_{\xi}$ and the topologies $\tau_{C_{\xi}}, \tau_{\xi}$ and $\tau_{\xi}^{<}$.

Claim 7. For every $1 \leq \xi<\omega_{1}, P_{\xi} \in \Pi_{\xi}^{0}\left(\tau_{C_{\xi}}\right)$.

Proof. We prove the statement by induction on $\xi$. For $\xi=1$ the set $P_{1}$ is a single point, which is clearly $\tau_{C_{1}}$-closed.

Let now $\xi \geq 2$ and suppose that $P_{\eta} \in \Pi_{\eta}^{0}\left(\tau_{C_{\eta}}\right)$ for every $\eta<\xi$. Then

$$
P_{\xi}=\bigcap_{m<\omega}\left\{x \in C_{\xi}: x(m, \cdot) \in C_{\xi_{m}} \backslash P_{\xi_{m}}\right\} .
$$

Since $\tau_{C_{\xi}}$ is the product of the topologies $\tau_{\xi_{\xi_{m}}}$ and $P_{\xi_{m}}$ is $\Pi_{\xi_{m}}^{0}\left(\tau_{C_{\xi_{m}}}\right)$ by the induction hypothesis, $P_{\xi}$ is the intersection of sets of additive class lower than $\xi$, so the statement follows.

Claim 8.

(i) For every $1 \leq \xi<\omega_{1}, P_{\xi} \subseteq C_{\xi}$ is nowhere dense closed, hence meager in the topology $\tau_{\xi}$.

(ii) For $\xi \geq 2, P_{\xi} \subseteq C_{\xi}$ is a dense $G_{\delta}$, hence residual in the topology $\tau_{\xi}^{<}$

Proof. We prove the two statements together, by induction on $\xi$. For $\xi=1, P_{1}$ is a single point, which is clearly closed and nowhere dense.

Let now $\xi \geq 2$ and suppose that (i) holds for every $\eta<\xi$. We prove (ii) for $\xi$.

By (4) and (5), we have

$$
P_{\xi}=C_{\xi} \backslash \bigcup_{m<\omega} U_{\xi, m} .
$$


By the induction hypothesis, $P_{\xi_{m}}$ is nowhere $\tau_{\xi_{m}}$-dense and closed for every $m<\omega$, so, since $\tau_{\xi}^{<}$is the product of the topologies $\tau_{\xi_{m}}, U_{\xi, m}$ is nowhere $\tau_{\xi_{m}}^{<}$-dense for every $m<\omega$. Also, $U_{\xi, m}$ is a finite intersection of $\tau_{\xi}^{<}$-open and $\tau_{\xi}^{<}$-closed sets, thus it is an $F_{\sigma}$ set in the topology $\tau_{\xi}^{<}$for every $m<\omega$. Hence (6) shows that $P_{\xi}$ is $\tau_{\xi}^{<}$-dense and $G_{\delta}$.

Consider now statement (i) for $\xi$. To obtain $\tau_{\xi}$, we made open every set on the right hand side of (6), so $P_{\xi}$ is closed. Again using the fact that $P_{\xi_{m}}$ is nowhere $\tau_{\xi_{m}}$-dense, we infer that $\bigcup_{m<\omega} U_{\xi, m}$ meets every $\tau_{\xi}^{<}$-open set, hence it is also $\tau_{\xi}$-dense, so $P_{\xi}$ is nowhere $\tau_{\xi}$-dense. This finishes the proof.

Claim 9. For every $1 \leq \xi<\omega_{1},\left.\tau_{\xi}\right|_{P_{\xi}}=\left.\tau_{\xi}^{<}\right|_{P_{\xi}}$.

Proof. By definition, $U_{\xi, N} \cap P_{\xi}=\emptyset(N<\omega)$, so the statement follows.

Claim 10. For $2 \leq \xi<\omega_{1}$, every basic $\tau_{\xi}^{<}$-open [basic $\tau_{\xi}$-open, resp.] subset $G$ of $C_{\xi}$ is in $\Sigma_{1}^{0}\left(\tau_{C_{\xi}}\right) \cup \Pi_{\eta}^{0}\left(\tau_{C_{\xi}}\right)\left[\Pi_{\xi}^{0}\left(\tau_{C_{\xi}}\right)\right.$, resp.] for some $\eta<\xi$ depending on $G$.

Proof. We prove the statements by induction on $\xi$. For $\xi=2, \tau_{2}^{<}=\tau_{C_{2}}$, so the basic $\tau_{2}^{<}$-open sets are $\Sigma_{1}^{0}\left(\tau_{C_{2}}\right)$, as stated. Every new basic open set of $\tau_{2}$ compared to $\tau_{2}^{<}$is a finite intersection of $\tau_{C_{2}}$-open and $\tau_{C_{2}}$-closed sets, so the basic $\tau_{2}$-open sets are indeed in $\Pi_{2}^{0}\left(\tau_{C_{2}}\right)$.

Take now $\xi \geq 3$ and suppose that the statements are true for every $\eta<\xi$. By (3) and the induction hypothesis, the $\tau_{\xi}^{<}$-open sets can be obtained as finite intersections of sets in $\Sigma_{1}^{0}\left(\tau_{C_{\xi}}\right) \cup \Pi_{\xi_{i}}^{0}\left(\tau_{C_{\xi}}\right)$ with $\xi_{i}<\xi$, which clearly gives sets in $\Pi_{\eta}^{0}\left(\tau_{C_{\xi}}\right)$ for $\eta<\xi$.

Consider now the topology $\tau_{\xi}$; again it is enough to determine the Borel class of the $U_{\xi, N}$ 's. By Claim 7, (4) shows that $U_{\xi, N}$ is a finite intersection of $\Sigma_{\xi_{i}}^{0}\left(\tau_{C_{\xi}}\right)$ and $\Pi_{\xi_{N}}^{0}\left(\tau_{C_{\xi}}\right)$ sets, which is a $\Pi_{\xi}^{0}\left(\tau_{C_{\xi}}\right)$ set, as required.

Claim 11. If $G$ is basic $\tau_{\xi}$-open and $G \cap P_{\xi} \neq \emptyset$ then $G$ is also basic $\tau_{\xi}^{<}$-open.

Proof. Since $U_{\xi, N}(N<\omega)$ is disjoint from $P_{\xi}$, the statement follows from the definition of $\tau_{\xi}$.

Claim 12. For every $1 \leq \xi<\omega_{1}$ and $N<\omega$,

$\left.\tau_{\xi}\right|_{U_{\xi, N}}=\operatorname{Pr}_{\prod_{i=0}^{N-1} C_{\xi_{i}}}\left(\left.\tau_{\xi}^{<}\right|_{\prod_{i=0}^{N-1} C_{\xi_{i}} \backslash P_{\xi_{i}}}\right) \times\left.\tau_{\xi_{N}}\right|_{P_{\xi_{N}}} \times \operatorname{Pr}_{\prod_{i=N+1}^{\infty} C_{\xi_{i}}}\left(\tau_{\xi}^{<}\right)$.

Proof. The sets $U_{\xi, N}(N<\omega)$ are pairwise disjoint, so

$$
\left.\tau_{\xi}\right|_{U_{\xi, N}}=\left.\tau_{\xi}^{<}\right|_{U_{\xi, N}} \text {. }
$$

By (3), $\operatorname{Pr}_{C_{\xi_{N}}}\left(\tau_{\xi}^{<}\right)=\tau_{\xi_{N}}$ while by $(4), \operatorname{Pr}_{C_{\xi_{N}}}\left(U_{\xi, N}\right)=P_{\xi_{N}}$ and

$$
\operatorname{Pr}_{\prod_{i=0}^{N-1} C_{\xi_{i}}}\left(U_{\xi, N}\right)=\prod_{i=0}^{N-1} C_{\xi_{i}} \backslash P_{\xi_{i}}, \quad \operatorname{Pr}_{\prod_{i=N+1}^{\infty} C_{\xi_{i}}}\left(U_{\xi, N}\right)=\prod_{i=N+1}^{\infty} C_{\xi_{i}},
$$


so we get

$$
\begin{aligned}
\left.\tau_{\xi}^{<}\right|_{U_{\xi, N}} & =\operatorname{Pr}_{\prod_{i=0}^{N-1} C_{\xi_{i}}}\left(\left.\tau_{\xi}^{<}\right|_{U_{\xi, N}}\right) \times \operatorname{Pr}_{C_{\xi_{N}}}\left(\left.\tau_{\xi}^{<}\right|_{U_{\xi, N}}\right) \times \operatorname{Pr}_{\prod_{i=N+1}^{\infty} C_{\xi_{i}}}\left(\left.\tau_{\xi}^{<}\right|_{U_{\xi, N}}\right) \\
& =\operatorname{Pr}_{\prod_{i=0}^{N-1} C_{\xi_{i}}}\left(\left.\tau_{\xi}^{<}\right|_{\prod_{i=0}^{N-1} C_{\xi_{i}} \backslash P_{\xi_{i}}}\right) \times\left.\tau_{\xi_{N}}\right|_{P_{\xi_{N}}} \times \operatorname{Pr}_{\prod_{i=N+1}^{\infty} C_{\xi_{i}}}\left(\tau_{\xi}^{<}\right),
\end{aligned}
$$

as required. This finishes the proof.

We will prove Theorem 4 through the following lemma, which states the same result in a more technical way, which fits better into an inductive argument.

Lemma 13. Fix $1 \leq \xi<\omega_{1}$. Let $(Z, \sigma)$ be an arbitrary Polish space and $G \subseteq Z \times C_{\xi}$ be a basic $\sigma \times \tau_{\xi}$-open set with

$$
G \cap\left(Z \times P_{\xi}\right) \neq \emptyset .
$$

(i) If $\xi \geq 2$ and $Q \subseteq Z \times C_{\xi}$ is $\Pi_{\vartheta}^{0}\left(\sigma \times \tau_{C_{\xi}}\right)$ for some $\vartheta<\xi$, and $Q \cap\left(Z \times P_{\xi}\right)$ is relatively $\sigma \times\left(\tau_{\xi} \mid P_{\xi}\right)$-residual in $G \cap\left(Z \times P_{\xi}\right)$, then $Q$ is $\sigma \times \tau_{\xi}$-residual in a $\sigma \times \tau_{\xi}$-open set $G^{\prime}$ satisfying

$$
G \cap\left(Z \times P_{\xi}\right)=G^{\prime} \cap\left(Z \times P_{\xi}\right) .
$$

(ii) If for a set $W \in \Sigma_{\xi}^{0}\left(\sigma \times \tau_{C_{\xi}}\right), W \cap\left(Z \times P_{\xi}\right)$ is relatively $\sigma \times\left(\tau_{\xi} \mid P_{\xi}\right)$ residual in $G \cap\left(Z \times P_{\xi}\right)$, then $W$ is $\sigma \times \tau_{\xi}$-residual in a $\sigma \times \tau_{\xi}$-open set $H \subseteq Z \times C_{\xi}$ such that $G \cap\left(Z \times P_{\xi}\right)$ is contained in the $\sigma \times \tau_{\xi}$-closure of $H$.

Proof. Once we prove the statement concerning $\Pi_{\vartheta}^{0}\left(\sigma \times \tau_{C_{\xi}}\right)$ sets for given $\vartheta<\xi<\omega_{1}$, the statement for $\Sigma_{\xi}^{0}\left(\sigma \times \tau_{C_{\xi}}\right)$ sets automatically follows. To see this, write

$$
W=\bigcup_{\alpha=0}^{\infty} Q_{\alpha},
$$

where $Q_{\alpha}$ is in $\Pi_{\vartheta_{\alpha}}^{0}\left(\sigma \times \tau_{C_{\xi}}\right)$ with $\vartheta_{\alpha}<\xi$ for every $\alpha<\omega$, and suppose that $W \cap G \cap\left(Z \times P_{\xi}\right)$ is relatively $\sigma \times\left(\tau_{\xi} \mid P_{\xi}\right)$-residual in $G \cap\left(Z \times P_{\xi}\right)$. For every $\alpha<\omega$, let $H_{\alpha}$ denote the maximal $\sigma \times \tau_{\xi}$-open set in which $Q_{\alpha}$ is $\sigma \times \tau_{\xi}$-residual. Then by (i), the $\sigma \times \tau_{\xi}$-open set $H=\bigcup_{\alpha=0}^{\infty} H_{\alpha}$ meets every open set intersecting $G \cap\left(Z \times P_{\xi}\right)$, which proves the statement.

So we need only prove (i). We do this by induction on $\xi$, namely we prove (i) for a fixed $\xi<\omega_{1}$ by assuming that (ii) holds for every $\eta<\xi$. For $\xi=1$, by the Baire Category Theorem, $H=W$ can be chosen.

Let now $\xi \geq 2$ and suppose that (ii) holds for every $\eta<\xi\left({ }^{1}\right)$. Consider a $\Pi_{\vartheta}^{0}\left(\sigma \times \tau_{C_{\xi}}\right)$ set $Q \subseteq Z \times C_{\xi}$ for some $\vartheta<\xi$ and suppose that $Q \cap\left(Z \times P_{\xi}\right)$ is $\sigma \times\left(\tau_{\xi} \mid P_{\xi}\right)$-residual in $G \cap\left(Z \times P_{\xi}\right)$ for a basic $\sigma \times \tau_{\xi}$-open set $G$ with $G \cap\left(Z \times P_{\xi}\right) \neq \emptyset$. By Claim 11, $G$ is in fact a basic $\sigma \times \tau_{\xi}^{<}$-open set.

$\left({ }^{1}\right)$ To be precise, we assume that the statements hold for every $\eta<\xi$ and Polish space $(Z, \sigma)$, no matter how we have fixed in (1) the sequence of ordinals $\eta_{i}(i<\omega)$ for every $\eta<\xi$. 
By Claim 9, the restrictions of the topologies $\tau_{\xi}$ and $\tau_{\xi}^{<}$to $P_{\xi}$ coincide, so $Q \cap G \cap\left(Z \times P_{\xi}\right)$ is also relatively $\sigma \times \tau_{\xi}^{<}$-residual in $G \cap\left(Z \times P_{\xi}\right)$. But by Claim $8\left(\right.$ ii),$P_{\xi}$ is a $\tau_{\xi}^{<}$-residual subset of $C_{\xi}$, so

$$
Q \cap G \text { is necessarily } \sigma \times \tau_{\xi}^{<} \text {-residual in } G \text {. }
$$

Let $0<I<\omega$ be minimal so that $\vartheta \leq \xi_{I}$. We show that

$$
G^{\prime}=G \cap\left(Z \times \prod_{i=0}^{I-1}\left(C_{\xi_{i}} \backslash P_{\xi_{i}}\right) \times C_{\xi_{I}} \times \prod_{i=I+1}^{\infty} C_{\xi_{i}}\right)
$$

meets the requirements. It is clearly $\sigma \times \tau_{\xi^{-}}$open and (7) holds, since

$$
Z \times P_{\xi} \subseteq Z \times \prod_{i=0}^{I-1}\left(C_{\xi_{i}} \backslash P_{\xi_{i}}\right) \times C_{\xi_{I}} \times \prod_{i=I+1}^{\infty} C_{\xi_{i}} .
$$

Suppose that $Q \cap G^{\prime}$ is not $\sigma \times \tau_{\xi^{-}}$-residual in $G^{\prime}$, that is, $Q \cap \widetilde{G}$ is $\sigma \times \tau_{\xi^{-}}$ meager for some basic $\sigma \times \tau_{\xi}$-open set $\widetilde{G} \subseteq G^{\prime}$; by passing to a proper basic $\sigma \times \tau_{\xi}$-open subset we can assume that $\widetilde{G}$ is not $\sigma \times \tau_{\xi}^{<}$-open. Let $\widetilde{G}_{0}$ be the basic $\sigma \times \tau_{\xi}^{<}$-open set such that

$$
\widetilde{G}=\widetilde{G}_{0} \cap\left(Z \times U_{\xi, J}\right)=\widetilde{G}_{0} \cap\left(Z \times \prod_{i=0}^{J-1} C_{\xi_{i}} \times P_{\xi_{J}} \times \prod_{i=J+1}^{\infty} C_{\xi_{i}}\right)
$$

for some $J<\omega$. This decomposition exists since $U_{\xi, N}(N<\omega)$ are pairwise disjoint. Note that $I \leq J$ by (9), and we have $\widetilde{G}_{0} \subseteq G$. To summarize, we have shown that

$$
Q \cap \widetilde{G}_{0} \cap\left(Z \times U_{\xi, J}\right) \text { is } \sigma \times \tau_{\xi} \text {-meager in } \widetilde{G}_{0} \cap\left(Z \times U_{\xi, J}\right) .
$$

Set

$$
\begin{aligned}
& \underline{Z}=Z \times \prod_{i=0}^{J-1} C_{\xi_{i}} \times \prod_{i=J+1}^{\infty} C_{\xi_{i}}, \\
& \underline{\sigma}=\sigma \times \operatorname{Pr}_{\prod_{i=0}^{J-1} C_{\xi_{i}}}\left(\tau_{\xi}^{<}\right) \times \operatorname{Pr}_{\prod_{i=J+1}^{\infty} C_{\xi_{i}}}\left(\tau_{\xi}^{<}\right) \\
& \underline{Q}=\left(Z \times C_{\xi}\right) \backslash Q \subseteq \underline{Z} \times C_{\xi_{J}}, \quad \underline{G}=\widetilde{G}_{0} \subseteq \underline{Z} \times C_{\xi_{J}} .
\end{aligned}
$$

The space $(\underline{Z}, \underline{\sigma})$ is clearly Polish, and $\underline{G}$ is a basic $\underline{\sigma} \times \tau_{\xi_{J}}$-open subset of $\underline{Z} \times C_{\xi_{J}}$. From $\vartheta \leq \xi_{I} \leq \xi_{J}<\xi, \underline{Q}$ is a $\Sigma_{\xi_{J}}^{0}\left(\underline{\sigma} \times \tau_{C_{\xi_{J}}}\right)$ set.

According to (10) and Claim $12, \underline{Q}$ is $\underline{\sigma} \times\left(\left.\tau_{\xi_{J}}\right|_{P_{\xi_{J}}}\right)$-residual in $\underline{G} \cap(\underline{Z} \times$ $\left.P_{\xi_{J}}\right)$, so by the induction hypothesis, $\underline{Q}$ is $\underline{\sigma} \times \tau_{\xi_{J}}$-residual in some $\underline{\sigma} \times$ $\tau_{\xi_{J}}$-open set $\underline{H} \subseteq \underline{Z} \times C_{\xi_{J}}$ such that the $\underline{\sigma} \times \tau_{\xi_{J}}$-closure of $\underline{H}$ contains $\underline{G} \cap\left(\underline{Z} \times P_{\xi_{J}}\right)$; so in particular, $\underline{H} \cap \underline{G} \neq \emptyset$. Let $H=\underline{H} \subseteq Z \times C_{\xi}$. Since $\tau_{\xi_{J}}=\operatorname{Pr}_{C_{\xi_{J}}}\left(\tau_{\xi}^{<}\right)$by definition, we have $H \cap \widetilde{G}_{0} \neq \emptyset$ and $Q \cap H \cap \widetilde{G}_{0}$ is $\sigma \times \tau_{\xi}^{<}$-meager in $H \cap \widetilde{G}_{0} \subseteq G$. This contradicts (8). The proof is complete. 
This lemma proves in particular that $P_{\xi}$ is a proper $\Pi_{\xi}^{0}\left(\tau_{C_{\xi}}\right)$ set.

Corollary 14. For every $1 \leq \xi<\omega_{1}, P_{\xi}$ is in $\Pi_{\xi}^{0}\left(\tau_{C_{\xi}}\right) \backslash \Sigma_{\xi}^{0}\left(\tau_{C_{\xi}}\right)$.

Proof. Clearly, $P_{\xi} \subseteq C_{\xi}$ is $\left.\tau_{\xi}\right|_{P_{\xi}}$-residual, but by Claim 8(i), $P_{\xi}$ is $\tau_{\xi^{-}}$ meager. So by Lemma $13, P_{\xi}$ cannot be $\Sigma_{\xi}^{0}\left(\tau_{C_{\xi}}\right)$.

Proof of Theorem 4. First we prove (i) and (ii) in the case $\xi=2$ since it is exceptional in Theorem 5 . Then we show (i) and (ii) for $3 \leq \xi<\omega_{1}$ and finally we treat the extension to all $2 \leq \xi<\omega_{1}$.

So let $\xi=2$. We set

$$
\begin{aligned}
U_{2, N}^{\prime} & =\left\{x \in 2^{\omega}: \forall n \geq N(x(n)=0)\right\} \quad(N<\omega), \\
P_{2} & =2^{\omega} \backslash \bigcup_{N<\omega} U_{2, N}^{\prime} .
\end{aligned}
$$

We define the topology $\tau_{2}$ as the refinement of $\tau_{C_{1}}$ by turning each point of the finite sets $U_{2, N}^{\prime}(N<\omega)$ into an open set. Clearly, $P_{2}$ is the complement of a dense countable subset in $\left(2^{\omega}, \tau_{C_{1}}\right)$, so in particular $P_{2}$ is $\Pi_{2}^{0}\left(\tau_{C_{1}}\right)$ and $\tau_{C_{1}}$-residual. Being the complement of the dense $\tau_{2}$-open set $\bigcup_{N<\omega} U_{2, N}^{\prime}$, it is also $\tau_{2}$-meager.

Let $A \subseteq X$ be $\Sigma_{2}^{0}(\tau)$ and take a continuous one-to-one mapping $\varphi$ : $\left(2^{\omega}, \tau_{C_{1}}\right)$ $\rightarrow(X, \tau)$ such that $\varphi^{-1}(A) \cap P_{2}$ is of second category in $P_{2}$ in the relative topology $\left.\tau_{2}\right|_{P_{2}}$. Then $\varphi^{-1}(A) \subseteq\left(2^{\omega}, \tau_{C_{1}}\right)$ is $\Sigma_{2}^{0}\left(\tau_{C_{1}}\right)$ and $\varphi^{-1}(A) \cap P_{2}$ is of second category in $\left.\tau_{2}\right|_{P_{2}}$; thus $\varphi^{-1}(A)$ is of second category in $\tau_{C_{1}}$ as well. Since a $\Sigma_{2}^{0}\left(\tau_{C_{1}}\right)$ set in $\left(2^{\omega}, \tau_{C_{1}}\right)$ is of second category only if its interior is nonempty, $\varphi^{-1}(A)$ contains a nonempty $\tau_{C_{1}}$-open set so $\varphi^{-1}(A) \cap U_{2, N}^{\prime} \neq \emptyset$ for some $N<\omega$. Then $\varphi^{-1}(A)$, having nonempty interior, is of second category in $\tau_{2}$, as required.

If $A$ is not $\Sigma_{2}^{0}(\tau)$, we apply Theorem 5 for $A_{0}=A, A_{1}=X \backslash A$. These sets cannot be separated by a $\Sigma_{\xi}^{0}(\tau)$ set, so since $P_{2}$ is the complement of a countable dense subset of $\left(2^{\omega}, \tau_{C_{1}}\right)$, there is a continuous one-to-one mapping $\varphi$ : $2^{\omega} \rightarrow X$ with $\varphi\left(P_{2}\right) \subseteq A, \varphi\left(2^{\omega} \backslash P_{2}\right) \subseteq X \backslash A$. So as we have seen above, $\varphi^{-1}(A)=P_{2}$ is indeed $\tau_{2}$-meager.

We turn to the $\xi \geq 3$ case. The Polish space $\left(C_{\xi}, \tau_{C_{\xi}}\right)$ is obviously homeomorphic to $\left(C_{1}, \tau_{C_{1}}\right)$ (see e.g. [2, Theorem 7.4, p. 35]). We show that $\left(P_{\xi}, \tau_{\xi}\right)$ satisfies the requirements for every $3 \leq \xi<\omega_{1}$.

Let $A \subseteq X$ be $\Sigma_{\xi}^{0}(\tau)$ for some $\xi<\omega_{1}$ and take a continuous one-to-one mapping $\varphi: C_{\xi} \rightarrow X$ such that $\varphi^{-1}(A) \cap P_{\xi}$ is of second category in $P_{\xi}$ in the relative topology $\left.\tau_{\xi}\right|_{P_{\xi}}$. Then $\varphi^{-1}(A) \subseteq C_{\xi}$ is $\Sigma_{\xi}^{0}\left(\tau_{C_{\xi}}\right)$ and $\varphi^{-1}(A) \cap P_{\xi}$ is $\left.\tau_{\xi}\right|_{P_{\xi}}$-residual in $G \cap P_{\xi}$ for some basic $\tau_{\xi}$-open set $G$. So according to Lemma $13(\mathrm{i}), \varphi^{-1}(A)$ is of second category in $\tau_{\xi}$, as required.

Suppose now that $A$ is not $\Sigma_{\xi}^{0}(\tau)$. We apply Theorem 5 for $A_{0}=A$ and $A_{1}=X \backslash A$. These sets cannot be separated by a $\Sigma_{\xi}^{0}(\tau)$ set, so since 
$P_{\xi}$ is $\Pi_{\xi}^{0}\left(\tau_{C_{\xi}}\right)$ but not $\Sigma_{\xi}^{0}\left(\tau_{C_{\xi}}\right)$, there is a continuous one-to-one mapping $\varphi: C_{\xi} \rightarrow X$ with $\varphi\left(P_{\xi}\right) \subseteq A, \varphi\left(2^{\omega} \backslash P_{\xi}\right) \subseteq X \backslash A$. So according to Claim 8.1(i), $\varphi^{-1}(A)=P_{\xi}$ is indeed $\tau_{\xi}$-meager.

Finally, suppose that for some cardinal $\lambda<2^{\aleph_{0}}$, in our model the union of $\lambda$ meager sets is meager in Polish spaces. Let $A_{i}(i<\lambda)$ be $\Sigma_{\xi}^{0}(\tau)$ for some $2 \leq \xi<\omega_{1}$ and set $A=\bigcup_{i<\lambda} A_{i}$. Since $\varphi^{-1}(A) \cap P_{\xi}$ is of second category in $P_{\xi}$ in the relative topology $\left.\tau_{\xi}\right|_{P_{\xi}}$, by our assumption $\varphi^{-1}\left(A_{i}\right) \cap P_{\xi}$ is also of second category in $\left(P_{\xi},\left.\tau_{\xi}\right|_{P_{\xi}}\right)$ for some $i<\lambda$. So by the first statement, $\varphi^{-1}\left(A_{i}\right) \subseteq \varphi^{-1}(A)$ is of second category in $\tau_{\xi}$. This finishes the proof.

Acknowledgments. The author is grateful to Gabriel Debs, Alain Louveau and Jean Saint Raymond for the helpful discussions.

\section{References}

[1] I. Juhász, Consistency results in topology, in: Handbook of Mathematical Logic, North-Holland, 1977, 503-522.

[2] A. S. Kechris, Classical Descriptive Set Theory, Grad. Texts in Math. 156, Springer, 1994.

[3] P. Komjáth, Limits of transfinite sequences of Baire-2 functions, Real Anal. Exchange 24 (1998/99), 497-502.

[4] A. Louveau and J. Saint Raymond, Borel classes and closed games: Wadge-type and Hurewicz-type results, Trans. Amer. Math. Soc. 304 (1987), 431-467.

[5] D. A. Martin and R. Solovay, Internal Cohen extensions, Ann. Math. Logic 2 (1970), 143-178.

[6] T. Natkaniec, The $\mathcal{J}$-almost constant convergence of sequences of real functions, Real Anal. Exchange 28 (2002/03), 481-491.

[7] W. Sierpiński, Sur les suites transfinies convergentes de fonctions de Baire, Fund. Math. 1 (1920), 132-141.

[8] S. Solecki, Decomposing Borel sets and functions and the structure of Baire class 1 functions, J. Amer. Math. Soc. 11 (1998), 521-550.

[9] J. Stern, Évaluation du rang de Borel de certains ensembles, C. R. Acad. Sci. Paris Sér. A-B 286 (1978), A855-A857.

Department of Analysis

Eötvös Loránd University

Pázmány Péter sétány 1/C

1117 Budapest, Hungary

E-mail: matrait@renyi.hu

Received 23 April 2004;

in revised form 20 July 2004 